\title{
Efficacy of Naldemedine for the Treatment of Opioid-Induced Constipation: A Meta-Analysis
}

\author{
Mohammad Esmadi $^{1}$, Dina Ahmad ${ }^{2}$, Alexander Hewlett ${ }^{3}$
}

1) Methodist Health System,

933 E Peirce St, Council Bluffs, Iowa 51503

2) Case Western Reserve University, 11100 Euclid Avenue, Cleveland, Ohio 44106

3) University of Nebraska Medical Center, 4400 Emile St., Omaha, NE 68105 USA

Address for correspondence: Dina Ahmad

Case Western Reserve University, 11100 Euclid Avenue, Cleveland, Ohio 44106, USA

dinaahmad@hotmail.com

Received: 10.08.2018

Accepted: 30.11 .2018

\section{ABSTRACT}

Background \& Aim: Opioid induced constipation (OIC) is the most common side effect of opioid therapy. It can lead to a decreased quality of life. Naldemedine is a peripherally acting $\mu$-opioid receptor antagonist that has been recently studied in randomized controlled trials (RCTs) for the management of OIC. The aim of this study is to perform a meta-analysis of existing clinical trials to estimate the efficacy and safety of naldemedine in opioid-induced constipation.

Methods: A systematic search of PubMed, CINAHL, Scopus, Cochrane database of systematic reviews, and ClinicalTrials.gov registry was performed in March 2018. Two independent reviewers systematically identified prospective RCTs published in the English language that compared the effect of oral naldemedine versus placebo in adults with OIC. Meta-analysis was performed using a random effects model to assess the primary outcome: spontaneous bowel movement (SBM) responder rates. Assessed secondary outcomes were: a change in SBM frequency per week from baseline during the treatment period, change from baseline in the frequency of complete SBM and incidence of treatment-emergent adverse events. Review Manager 5.3 software program was utilized for statistical analysis.

Results: Six RCTs met the inclusion criteria. A total of 2,762 patients were included in the meta-analysis. The proportion of SBM responders was significantly higher in the naldemedine group compared to the placebo group ( $56.4 \%$, vs. $34.7 \%$, p $<0.00001)$. There was no statistically significant difference in treatment-emergent adverse events between naldemedine group and placebo group (mean odds ratio $=1.18, \mathrm{p}=0.25,95 \% \mathrm{CI}$ : $0.89-1.55)$. Change in SBM frequency was higher in the naldemedine group versus placebo group $(\mathrm{p}<0.00001)$, as well as the change in complete SBM frequency.

Conclusions: Naldemedine $0.2 \mathrm{mg}$ daily significantly improved symptoms in patients with opioid-induced constipation and was generally well tolerated. These results support the use of naldemedine for the treatment of opioid-induced constipation.

Key words: Naldemedine - opioid - constipation - opioid-induced constipation.

Abbreviation: AE: adverse event; BSS: Bristol Stool Scale; CNS: central nervous system; CSBM: complete spontaneous bowel movement; GI: gastrointestinal; OIC: opioid induced constipation; PAMORA: peripherally acting $\mu$-opioid receptor antagonists; P-gp: P-glycoprotein; RCT: randomized controlled trial; SBM: spontaneous bowel movement.

\section{INTRODUCTION}

Chronic pain is common in the United States adults with an estimated prevalence of around $30.7 \%$ [1]. Opioids are commonly used in the treatment of pain related to many cancer and non-cancer illnesses. Around 40-47 per 1,000 individuals (approximately 13-15 million people in total) use opioids in the US [2]. Opioid induced constipation (OIC) is the most common side effect of opioids [3]. In a population-based survey, OIC was reported by $57 \%$ of the patients with chronic non-cancer pain who used opioids [4], leading to a significant impact on the patients' well-being. Health-related quality of life is significantly worse in patients with OIC compared to patients without OIC. This was demonstrated in a survey of 359 patients using opioids [5]. Furthermore, it does not appear that patients develop tolerance to OIC [6]. 
Opioids relieve pain by activating opioid receptors in the central and peripheral nervous systems. Opioid $\mu$ receptors are present throughout the gastrointestinal (GI) tract; when opioids bind to them, the neural activity in the submucosal and myenteric plexuses of the enteric nervous system decrease. This results in the impairment of the GI transit and motility, a decrease in gut fluid secretion, and an increase in fluid absorption, which can lead to opioid induced constipation [3].

Laxatives are usually used as first line treatment option for OIC. However, laxatives do not target the underlying mechanism of OIC. There is lack of evidence regarding the efficacy of laxatives in the treatment of OIC. In a Cochrane systematic review on management of OIC in patients with advanced illness, four randomized trials compared different kinds of laxatives and showed no significant differences among them [7].

Currently available treatment options for OIC include peripherally acting $\mu$-opioid receptor antagonists (PAMORA, including methylnaltrexone, naloxegol, naldemedine, and alvimopan), lubiprostone (a type-2 chloride channel activator that induces secretion of fluid in the intestine) and oral naloxone [8].

Naldemedine is an orally active PAMORA that was approved in the United States for OIC in adult patients with chronic non-cancer pain [9]. Benefit for OIC in non-cancer patients was shown in two identically designed, double-blind, placebo-controlled, 12-week phase III trials conducted in patients with non-cancer chronic pain and OIC (COMPOSE I and II) [10].

The efficacy of naldemedine was also shown in the treatment of cancer-related OIC in a randomized, placebocontrolled, phase III trial where $71 \%$ responded to $0.2 \mathrm{mg} /$ day compared with $34 \%$ of the placebo group, using the same response criteria as used in the COMPOSE trials [11], and the drug can be used off-label in cancer patients.

More trials investigated naldemedine and the aim of this study is to review the evidence from randomized controlled trials (RCTs) related to the efficacy of Naldemedine.

\section{METHODS}

\section{Literature Search}

We searched the electronic literature from ClinicalTrials. gov, MEDLINE/PubMed, CINAHL, Scopus, MetaRegister of Controlled Trials, Database of Abstracts of Reviews of Effects (DARE), Cochrane Central Register of Controlled Trials, and Cochrane Database of Systematic Reviews in March 2018. We used the key terms naldemedine and opioid induced constipation. We also conducted manual searches of reference lists from relevant papers in order to identify any other additional articles. We searched all relevant articles regardless of document type or results. We excluded trials published in non-English language and trials conducted in animals.

\section{Study Design}

Two investigators (D.A. and M.E.) reviewed independently the titles and abstracts of all the citations that were identified in our literature search. We selected all randomized placebo- controlled clinical trials of naldemedine in treatment of OIC. We used the following inclusion criteria: (1) studies that examined the effect of naldemedine on symptoms of OIC, (2) studies that were randomized, prospective and placebo controlled published in peer-reviewed journals, and (3) studies in humans. We excluded retrospective analyses, abstracts, review articles and case reports.

\section{Data extraction and risk of bias}

Two of the authors (M.E., D.A.) extracted data from eligible studies independently. A common data extraction form was used with any disagreements in the data resolved by consensus between authors. Only articles that met the inclusion criteria mentioned above were selected.

The risk of bias in RCTs was assessed using a modified version of the Cochrane Collaboration's tool for assessing risk of bias. Trials were analyzed across domains that are subject to potential bias, including sequence generation, allocation concealment, blinding, baseline imbalance, incomplete outcome data, selective outcome reporting, and other sources of bias for the RCTs. The overall risk of bias for each study was considered low if the risk of bias was low in all domains, high if the risk of bias was high in at least one domain, or moderate if the risk of bias was moderate in at least one domain (with no domains having a high risk of bias). Bias assessment was performed by two authors (M.E. and D.A.) with disagreements resolved by consensus.

\section{Assessment of Outcomes}

The primary outcome assessed was spontaneous bowel movement (SBM) responder rates (a responder had at least three SBMs per week with an increase from baseline of at least one SBM per week). The secondary outcomes were: change in SBM frequency per week from baseline during the treatment period, change from baseline in the frequency of complete SBM (CSBM was defined as an SBM with the feeling of complete evacuation), and the incidence of treatment-emergent adverse events (AE).

\section{Statistical Analysis}

A meta-analysis was performed comparing the efficacy of naldemedine to no naldemedine or placebo for treatment of OIC by calculating pooled estimates of the primary outcome of SBM responder rates, and secondary outcomes of change from baseline in SBM frequency per week, change from baseline in the frequency of CSBM, and frequency of AEs using odds ratio (OR) with random effects model. Statistical significance was observed if $\mathrm{p}<0.05$ or range in the confidence interval did not include 1. Funnel plots were used to assess publication bias. Heterogeneity among studies was assessed by calculating the $\mathrm{I}^{2}$ measure of inconsistency, which was considered significant if $\mathrm{p}<0.10$ or $\mathrm{I}^{2}>50 \%$. Statistical analysis was performed by the software RevMan 5.3.

\section{RESULTS}

Initial search resulted in 898 articles and abstracts. Of the 898 citations identified, we excluded 882 after screening the titles and abstracts, leaving 16 articles for full-text review. Of 


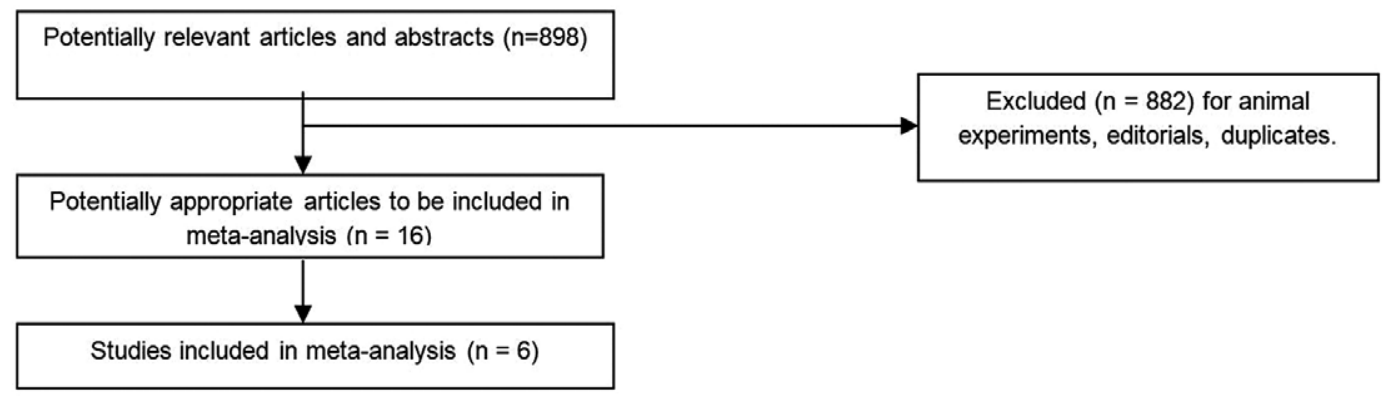

Fig. 1. Identification of eligible randomized controlled trials.

the 16 articles remaining, 6 articles met the inclusion criteria $(\mathrm{n}=2,762)$ and were included in the meta-analysis (Fig. 1).

Two RCTs were performed in Japan, one performed in Japan and Korea and three were performed in multiple countries. They were published between 2017 and 2018 in English. Of the six studies, five investigated the primary outcome of SBM responder rate [10-13] and all studies investigated the secondary outcome of frequency of AE [10-14]. All of the studies assessed the dose of $0.2 \mathrm{mg}$, but two studies assessed $0.1 \mathrm{mg}$ and $0.4 \mathrm{mg}$ doses as well [12-13]. We only investigated the $0.2 \mathrm{mg}$ dose outcomes as there is not enough data to study the 0.1 and $0.4 \mathrm{mg}$ doses outcomes. Table I shows the basic characteristics of the included studies.

The proportion of SBM responders was significantly higher with the naldemedine group versus the placebo group $(56.4 \%$ vs. $34.7 \%, p<0.00001)$. There was no statistically significant difference in treatment-emergent AEs between naldemedine group and placebo group with a mean odds ratio of $1.18, \mathrm{p}$ $=0.25$ (95\% CI: 0.89-1.55). Change in SBM frequency was higher in the naldemedine group versus the placebo group $(\mathrm{p}<0.00001)$, as well as the change in CSBM frequency $(\mathrm{p}<0.00001)$ (Fig. 2).

The identified AEs were: diarrhea, abdominal pain, vomiting, decreased appetite, decreased white blood cells count, nasopharyngitis, decreased total protein, hypertension, increased blood alkaline phosphatase and increased blood lactate dehydrogenase. Diarrhea was the most common.

Bristol Stool Scale (BSS) was not used in all studies. Katakami et al. [12] identified diarrhea as an AE if patients reported BSS $>7$. Katakami et al. [11] in COMPOSE-4 study used BSS 1 or 2 in the inclusion criteria. Webster et al. [13] used BSS $<3$ in the inclusion criteria.

Funding of RCTs was mainly by Shionogi \& Co, a Japanese pharmaceutical company, in addition to other pharmaceutical companies.

\section{Risk of bias in the included studies}

The risk of bias in the included RCTs was low. All included studies reported clearly the utilization of randomization and allocation methods that minimized the risk of bias apart from Katakami et al. [12]. All studies were double-blinded. No significant baseline imbalances, missing outcome data or selective outcome reporting were noted. Details of risk of bias assessment are summarized in Table II. No evidence of publication bias was observed by visual inspection of the funnel plot (Fig. 3). However, this assessment cannot prove the absence of bias due to the small number of studies.

\section{DISCUSSION}

As the use of narcotics continues to be prevalent, OIC remains a challenging side effect that can significantly affect patients' quality of life. Naldemedine is a novel medication that appears to have a favorable outcome for OIC. In addition to being approved in the United States for adult non-cancer patients, the European Medicines Agency (EMA) has approved naldemedine for treatment of OIC without the restriction to non-cancer patients [15].

Table I. Characteristics of studies included in the meta-analysis. RCT: randomized controlled trials.

\begin{tabular}{|c|c|c|c|c|c|c|c|}
\hline Author and year & Location & Type & $\begin{array}{l}\text { No. } \\
\text { Patients }\end{array}$ & $\begin{array}{l}\text { Gender } \\
(\mathrm{M} / \mathrm{F})\end{array}$ & $\begin{array}{l}\text { Mean or } \\
\text { median age }\end{array}$ & Duration & Naldemedine dose \\
\hline Hale et al. 2017 & Multiple & RCT & 545 & $216 / 329$ & 53 & 12 weeks & Naldemedine $0.2 \mathrm{mg}$ daily \\
\hline \multirow[t]{2}{*}{ Hale et al. 2017} & Multiple & RCT & 550 & $217 / 333$ & 54 & 12 weeks & Naldemedine $0.2 \mathrm{mg}$ daily \\
\hline & & & & & & & Naldemedine $0.1 \mathrm{mg}$ daily \\
\hline \multirow[t]{3}{*}{ Katakami et al. 2017} & Japan, Korea & RCT & 225 & $134 / 91$ & 64.2 & 2 weeks & Naldemedine $0.2 \mathrm{mg}$ daily \\
\hline & & & & & & & Naldemedine $0.4 \mathrm{mg}$ daily \\
\hline & & & & & & & Naldemedine $0.2 \mathrm{mg}$ daily \\
\hline $\begin{array}{l}\text { Katakami et al. } 2017 \\
\text { (COMPOSE 4) }\end{array}$ & Japan & RCT & 193 & $119 / 74$ & 64.2 & 2 weeks & Naldemedine $0.1 \mathrm{mg}$ daily \\
\hline \multirow[t]{2}{*}{ Webster et al. 2017} & Japan & RCT & 238 & $71 / 167$ & 51.8 & 4 weeks & Naldemedine $0.2 \mathrm{mg}$ daily \\
\hline & & & & & & & Naldemedine $0.4 \mathrm{mg}$ daily \\
\hline Webster et al. 2018 & Multiple & RCT & 1240 & $455 / 785$ & 53 & 52 weeks & Naldemedine $0.2 \mathrm{mg}$ daily \\
\hline
\end{tabular}


SBM responders

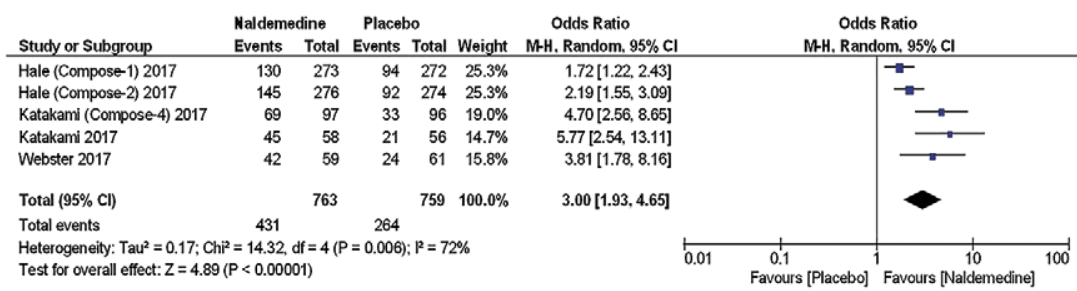

Change in SBM frequency from baseline

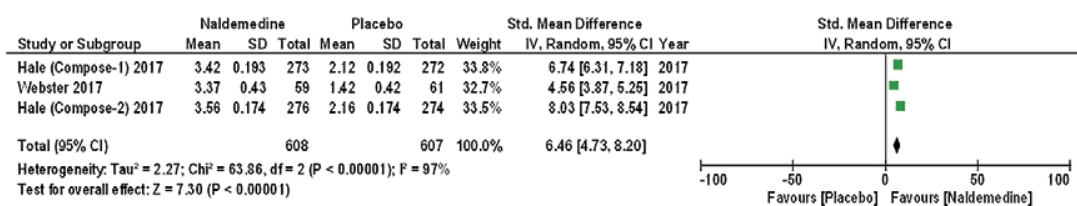

Change in CSBM frequency from baseline

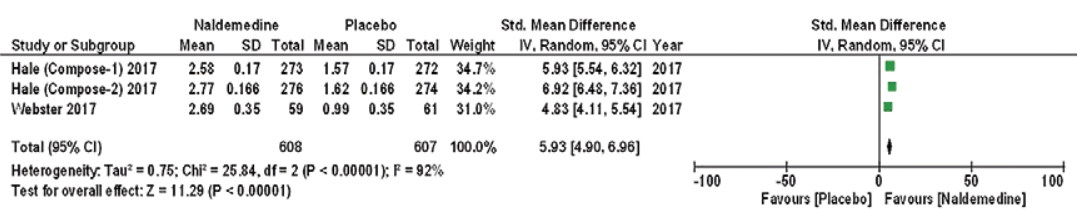

Treatment-emergent adverse events:

\begin{tabular}{|c|c|c|c|c|c|c|c|}
\hline \multirow[b]{2}{*}{ Study or Subgroup } & \multicolumn{2}{|c|}{ Naldemedine } & \multicolumn{2}{|c|}{ Placebo } & \multirow[b]{2}{*}{ Weight } & \multirow{2}{*}{$\begin{array}{l}\text { Odds Ratio } \\
\text { M-H, Random, } 95 \% \mathrm{Cl} \text { Year }\end{array}$} & \multirow{2}{*}{$\begin{array}{c}\text { Odds Ratio } \\
\text { M.H, Random, } 95 \% \mathrm{CI}\end{array}$} \\
\hline & Events & Total & & Total & & & \\
\hline Hale (Compose-1) 2017 & 132 & 271 & 123 & 272 & $21.4 \%$ & $1.15[0.82,1.61] 2017$ & \\
\hline Katakami (Compose-4) 2017 & 43 & 97 & 25 & 96 & $12.4 \%$ & $2.26[1.23,4.15] \quad 2017$ & $\longrightarrow$ \\
\hline Hale (Compose-2) 2017 & 136 & 271 & 132 & 274 & $21.5 \%$ & $1.08[0.77,1.52] 2017$ & $=$ \\
\hline Webster 2017 & 30 & 60 & 31 & 61 & $10.1 \%$ & $0.97[0.47,1.97] 2017$ & \\
\hline Katakami 2017 & 39 & 58 & 29 & 56 & $9.3 \%$ & $1.91[0.90,4.08] 2017$ & \\
\hline Webster 2018 & 425 & 621 & 446 & 619 & $25.3 \%$ & $0.84[0.66,1.07] 2018$ & $=$ \\
\hline Total $(95 \% \mathrm{Cl})$ & & 1378 & & 1378 & $100.0 \%$ & $1.18[0.89,1.55]$ & 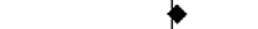 \\
\hline Total events & 805 & & 786 & & & & \\
\hline $\begin{array}{l}\text { Heterogeneity: } \text { Tau }^{2}=0.06 ; C \\
\text { Test for overall effect: } Z=1.15\end{array}$ & $\begin{array}{l}=12.07, \\
P=0.25)\end{array}$ & & & $F^{2}=59$ & & & $0.1{ }^{1}{ }^{1}{ }^{1}$ lacebo] [Na \\
\hline
\end{tabular}

Fig. 2. Forest plot demonstrating primary and secondary outcomes of naldemedine compared to placebo. SBM: small bowel movements; CSBM: complete small bowel movements.

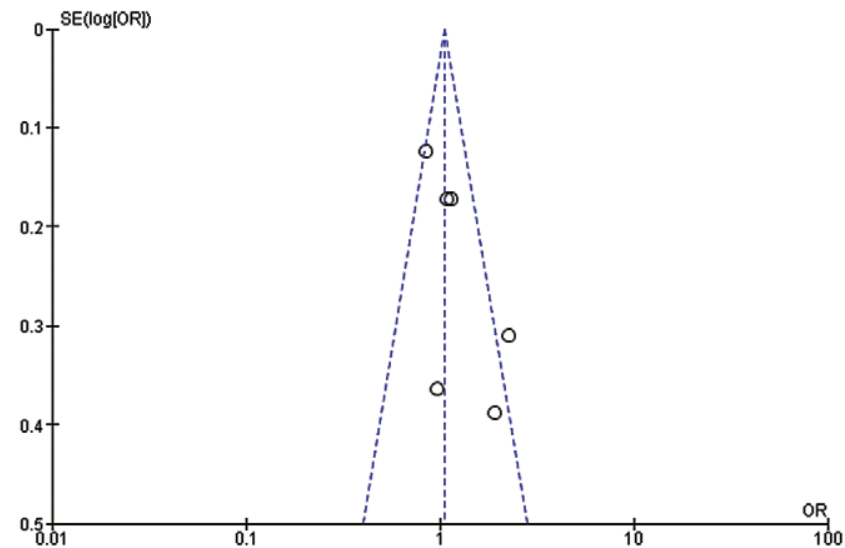

Fig. 3. Funnel plot showing no publication bias.

The $\mu$, delta $(\delta)$-, and kappa $(\kappa)$-opioid receptors are present in the central nervous system (CNS) and the GI tract as well. While the $\delta$ - and $\kappa$-receptors are mainly found in the proximal colon and stomach, the $\mu$-receptors are widely present throughout the GI tract. Opioid induced constipation is mainly a result of bowel $\mu$-opioid receptor activation [16]. Naldemedine binds to the $\mu$-, $\delta$-, and $\kappa$-opioid receptors and decreases the constipating effects of opioids through its action mainly as an antagonist at the $\mu$-opioid receptors in GI tract $[17,18]$. Structurally, naldemedine is similar to naltrexone with an additional side chain leading to increased molecular weight and polar surface area [18]. Naldemedine also is a substrate of the P-glycoprotein (P-gp) efflux transporter. These properties makes it less possible for naldemedine to interfere with the central effect of opioids by decreasing its penetration into the CNS [17].

If used with strong hepatic cytochrome P450 3A (CYP3A) inducers, such as phenytoin, carbamazepine, rifampin, and St. John's Wort, the plasma concentrations of naldemedine can be reduced, which may decrease its efficacy. An increase in plasma concentrations of naldemedine can happen if naldemedine is administered with moderate or strong CYP3A inhibitors or P-gp inhibitors, such as quinidine amiodarone, cyclosporine, captopril, quercetin, and verapamil. Potentially, naldemedine can augment opioid receptor antagonism and increase opioid withdrawal if given concurrently with other opioid antagonists [17].

The five RCTs we analyzed used $0.2 \mathrm{mg}$ dose. In Katakami et al. [12] the dose of $0.2 \mathrm{mg}$ provided the most favorable safety and efficacy profile. All three doses $(0.1,0.2$ and 0.4 
Table II. Risk of bias assessment of all randomized controlled trials.

\begin{tabular}{lcccccc}
\hline Study & $\begin{array}{c}\text { Random } \\
\text { Sequence } \\
\text { Generation }\end{array}$ & $\begin{array}{c}\text { Allocation } \\
\text { concealment }\end{array}$ & $\begin{array}{c}\text { Blinding of } \\
\text { Participants or } \\
\text { Personnel }\end{array}$ & $\begin{array}{c}\text { Blinding of } \\
\text { Outcome } \\
\text { Assessment }\end{array}$ & $\begin{array}{c}\text { Incomplete } \\
\text { Outcome Data }\end{array}$ & $\begin{array}{c}\text { Selective } \\
\text { Reporting / } \\
\text { Reporting Bias }\end{array}$ \\
\hline Hale 2017 & Yes & Yes & Yes & Unclear & No & None Bias \\
Katakami 2017 & Unclear & Unclear & Yes & Yes & No & None \\
$\begin{array}{l}\text { Katakami 2017 } \\
\text { (COMPOSE-4) }\end{array}$ & Yes & Yes & Yes & Unclear & No & None \\
Webster 2017 & Yes & Unclear & Yes & Unclear & No & None \\
Webster 2018 & Yes & Yes & Yes & No & No & None \\
\hline
\end{tabular}

mg) were generally well tolerated; however, the incidence of AEs increased in a dose-dependent fashion. Naldemedine $0.4 \mathrm{mg}$ was associated with a significantly greater incidence of AEs compared with placebo. Naldemedine $0.1 \mathrm{mg}$ did not show significant improvements versus placebo although it had the lowest incidence of AEs. The $0.2 \mathrm{mg}$ dose showed significant improvements in all efficacy assessments and did not substantially increase the incidence of AEs compared with the placebo.

In a study conducted by Webster et al. [13], naldemedine $0.2 \mathrm{mg}$ or $0.4 \mathrm{mg}$ significantly increased the frequency of weekly SBMs (primary end point) from baseline compared to the placebo. Change in the frequency of weekly SBMs in the naldemedine $0.1 \mathrm{mg}$ group was not significantly different from that in the placebo group. Naldemedine $0.4 \mathrm{mg}$ produced a slightly greater increase in mean weekly SBM frequency than naldemedine $0.2 \mathrm{mg}$, but the difference was not significant, suggesting no increase in efficacy with a higher dose. The 0.1 mg dose did not show significant improvement in primary or secondary outcomes. The incidence of treatment-related AEs increased with the naldemedine dose. The highest number of treatment related AEs was reported in patients treated with naldemedine $0.4 \mathrm{mg}$. This suggests a dose-related response, with a better safety profile being demonstrated for naldemedine $0.2 \mathrm{mg}$ than for naldemedine $0.4 \mathrm{mg}$.

Based on the overall efficacy and safety profiles demonstrated, Katatami et al. [12] and Webster et al. [13] chose naldemedine $0.2 \mathrm{mg}$ for their subsequent RCTs $[11,14]$.

Our meta-analysis of six RCTs demonstrated that naldemedine $0.2 \mathrm{mg}$ did a statistically significant improvement in the primary and secondary outcomes as compared to a placebo. It also demonstrated that there was no statistically significant difference in adverse outcomes with naldemedine over placebo. Naldemedine not only increases the frequency of SBMs but also increases the frequency of CSMBs, which is important for symptomatic relief in OIC.

There are multiple strengths to this meta-analysis. We performed a comprehensive search of the literature, which allowed for the maximum number of relevant studies to be involved in the meta-analysis. All studies used in the metaanalysis were RCTs, which maximized the relevancy of the studies. Five out of the six studies reached the primary endpoint, maximizing the significance of the study. Moreover, no publication bias was noted.

There are some limitations of this meta-analysis. Of the six studies done, a total of $43 \%$ of patients reached the primary end point, leading to a smaller number of patients maximized in the studies. Two authors conducted two studies each, which may introduce bias. The RCTs varied in time for which patients were followed, ranging from 2 weeks to 52 weeks. In Hale et al. study [10], two parallel trials were presented with similar design and presented in one manuscript. We counted them as two different studies. Finally, Webster et al. [14] used adverse effects as a primary outcome and was the longest in duration (52 weeks). The rest of the studies were 2 to 12 weeks in duration. It is possible that those short-duration studies missed certain AEs due to the short observation time.

There are currently no other ongoing trials registered on ClinicalTrials.gov investigating naldemedine. More trials investigating naldemedine will strengthen the body of evidence regarding its efficacy and safety. Also, there are no studies so far that have compared naldemedine head-to-head with other agents. Comparing naldemedine to other PAMORAs will be helpful.

\section{CONCLUSION}

Naldemedine $0.2 \mathrm{mg}$ significantly improved symptoms in patients with opioid-induced constipation and was generally well tolerated. These results support the use of naldemedine for the treatment of opioid-induced constipation.

Conflicts of interest: The authors deny any conflict of interest.

Authors' contributions: E.M. and E.M.: acquisition of data, analysis and interpretation of data; E.M.: drafting the article; A.D.: design of the study; H.A.: critical revision and final approval.

\section{REFERENCES}

1. Johannes CB, Le TK, Zhou X, Johnston JA, Dworkin RH. The prevalence of chronic pain in United States adults: Results of an Internet-based survey. J Pain 2010;11:1230-1239. doi:10.1016/j.jpain.2010.07.002

2. Boudreau D, Von Korff M, Rutter CM, et al. Trends in long-term opioid therapy for chronic non-cancer pain. Pharmacoepidemiol Drug Saf 2009;18:1166-1175. doi:10.1002/pds.1833

3. Camilleri M, Drossman DA, Becker G, et al. Emerging treatments in neurogastroenterology: A multidisciplinary working group consensus statement on opioid-induced constipation. Neurogastroenterol Motil 2014;26:1386-1395. doi:10.1111/nmo.12417

4. Cook SF, Lanza L, Zhou X, et al. Gastrointestinal side effects in chronic opioid users: Results from a population-based survey. Aliment Pharmacol Ther 2008;27:1224-1232. doi:10.1111/j.13652036.2008.03689.x 
5. Bell T, Annunziata K, Leslie JB. Opioid-induced constipation negatively impacts pain management, productivity, and health-related quality of life: Findings from the National Health and Wellness Survey. J Opioid Manag 2009;5:137-144.

6. Gyawali B, Hayashi N, Tsukuura H, Honda K, Shimokata T, Ando Y Opioid-induced constipation. Scand J Gastroenterol 2015;50:1331-1338. doi:10.3109/00365521.2015.1054423

7. Candy B, Jones L, Larkin PJ, Vickerstaff V, Tookman A, Stone P. Laxatives for the management of constipation in people receiving palliative care. Cochrane Database Syst Rev 2015;(5):CD003448. doi:10.1002/14651858.CD003448.pub4

8. Sridharan K, Sivaramakrishnan G. Drugs for Treating Opioid-Induced Constipation: A Mixed Treatment Comparison Network Metaanalysis of Randomized Controlled Clinical Trials. J Pain Symptom Manag 2018;55:468-479.e1. doi:10.1016/j.jpainsymman.2017.08.022

9. Food and Drug Administration. NDA 208854. Accessed on June 30, 2018. Available at: https://www.accessdata.fda.gov/drugsatfda_docs/ap pletter/2017/208854Orig1s000ltr.pdf

10. Hale M, Wild J, Reddy J, Yamada T, Arjona Ferreira JC. Naldemedine versus placebo for opioid-induced constipation (COMPOSE-1 and COMPOSE-2): two multicentre, phase 3, double-blind, randomised, parallel-group trials. Lancet Gastroenterol Hepatol 2017;2:555-564. doi:10.1016/S2468-1253(17)30105-X

11. Fatakami N, Harada T, Murata T, et al. Randomized Phase III and Extension Studies of Naldemedine in Patients With Opioid-Induced Constipation and Cancer. J Clin Oncol 2017;35:3859-3866. doi:10.1200/JCO.2017.73.0853
12. Katakami N, Oda K, Tauchi K, et al. Phase IIb, Randomized, DoubleBlind, Placebo-Controlled Study of Naldemedine for the Treatment of Opioid-Induced Constipation in Patients With Cancer. J Clin Oncol 2017;35:1921-1928. doi:10.1200/JCO.2016.70.8453

13. Webster LR, Yamada T, Arjona Ferreira JC. A Phase 2b, Randomized, Double-Blind Placebo-Controlled Study to Evaluate the Efficacy and Safety of Naldemedine for the Treatment of Opioid-Induced Constipation in Patients with Chronic Noncancer Pain. Pain Med 2017;18:2350-2360. doi:10.1093/pm/pnw325

14. Webster LR, Nalamachu S, Morlion B, Reddy J, Baba Y, Yamada T, Arjona Ferreira JC. Long-term use of naldemedine in the treatment of opioid-induced constipation in patients with chronic non-cancer pain: a randomized, double-blind, placebo-controlled phase 3 study. Pain 2018;159:987-994. doi:10.1097/j.pain.0000000000001174

15. http://www.ema.europa.eu/ema/index.jsp?curl=pages/ medicines/pips/EMEA-001893-PIP01-15/pip_001533. jsp\&mid=WC0b01ac058001d129 (Accessed on October 30, 2018).

16. Camilleri M, Drossman DA, Becker G, Webster LR, Davies AN, Mawe GM. Emerging treatments in neurogastroenterology: a multidisciplinary working group consensus statement on opioidinduced constipation. Neurogastroenterol Motil 2014;26:1386-1395. doi:10.1111/nmo.12417

17. Symproic (naldemedine) prescribing information. Florham Park, NJ: Shionogi Inc; March 2017.

18. Markham A. Naldemedine: first global approval. Drugs 2017;77:923927. doi:10.1007/s40265-017-0750-0 